\title{
One-Year Follow-Up of Serum Prolactin Level in Schizophrenia Patients Treated with Blonanserin: A Case Series
}

\author{
Sakae Takahashi ${ }^{\bowtie}$, Masahiro Suzuki, and Makoto Uchiyama \\ Division of Psychiatry, Department of Psychiatry, Nihon University, School of Medicine, Tokyo, Japan
}

\begin{abstract}
In our previous study, a prolactin elevation was more frequently in risperidone than in blonanserin; however, it was more often in blonanserin than in olanzapine. Therefore, while a rate of PRL rising is low to moderate, hyperprolactinemia is a considerable adverse effect in the blonanserin treatment. In this study, to examine detailed characteristics of hyperprolactinemia of blonanserin, we analyzed the prolactin data in six schizophrenic patients who were switched to blonanserin from other antipsychotics and followed for one year. As a result, blonanserin dose was clearly associated with serum prolactin level. The average prolactin level was almost normal when the mean blonanserin dosage was $8.0 \mathrm{mg} /$ day. Regardless of the dose decrease of blonanserin, there were no remarkable changes in symptoms and social functions. Based on our findings, we conclude that low dose blonanserin medication may be useful for schizophrenia maintenance treatment without hyperprolactinemia and a high rate of relapse.

Psychiatry Investig 2015;12(4):566-568
\end{abstract}

Key Words Blonanserin, Dose dependency, Hyperprolactinemia, Maintenance treatment, Schizophrenia.

\section{INTRODUCTION}

Blonanserin (BNS) is a relatively new atypical antipsychotic drug, and has been used in Korea and Japan for approximately three and a half, and six years, respectively. BNS has a high affinity for $\mathrm{D}_{2,3}$ and $5-\mathrm{HT}_{2 \mathrm{~A}}$ receptors. The affinity for $\mathrm{D}_{2}$ is several-fold higher than that for $5-\mathrm{HT}_{2 \mathrm{~A}} \cdot{ }^{1}$ We have reported a follow-up study of psychotic patients treated with BNS. ${ }^{2}$ In our study, a prolactin (PRL) elevation was more frequently in risperidone (RIS) than in BNS. A weight gain is more common in olanzapine (OLZ) than in BNS. Furthermore, there were no differences between BNS and other antipsychotics in the extrapyramidal symptom (EPS) rate. Consequently, we concluded that BNS might be useful for the maintenance treatment of schizophrenia without inducing hyperprolactinemia and weight gain. However, our study also showed that hyperprolactinemia was more often in BNS than in OLZ. Hence, although a rate of PRL elevation is low to moderate,

Received: October 3, 2014 Revised: March 2, 2015

Accepted: March 16, 2015 Available online: September 30, 2015

$\triangle$ Correspondence: Sakae Takahashi, MD, PhD

Department of Neuropsychiatry, Nihon University, School of Medicine, 30-1 Oyaguchi, Kamicho, Itabashi-ku, Tokyo 173-8610, Japan

Tel: +81-3-3972-8111, Fax: +81-3-3974-2920

E-mail: takahashi.sakae@nihon-u.ac.jp

(a) This is an Open Access article distributed under the terms of the Creative Commons Attribution Non-Commercial License (http://creativecommons.org/licenses/bync/3.0) which permits unrestricted non-commercial use, distribution, and reproduction in any medium, provided the original work is properly cited. hyperprolactinemia is a considerable adverse effect in the BNS treatment. This was also reported by other previous studies. ${ }^{3}$

In the above our study, we tried to follow 10 patients for one year; however, four of the 10 cases discontinued the BNS treatment. Two of the four cases stopped it due to elevated PRL; other two cases discontinued it due to relapses. In this study, to examine more detailed characteristics of hyperprolactinemia of BNS, we closely reanalyzed the PRL data in the six cases that we were able to follow for one year. However, in the six cases, one case had mental retardation. BNS is an antipsychotic drug for schizophrenia. Therefore, in order to establish biological homogeneity, we removed this case. Instead, a new schizophrenia case was added in this study.

\section{CASE}

Subjects were six pre-menopausal female schizophrenic patients (five schizophrenics were used in our past study; one was additional case), with a mean age of $36.2 \pm 9.2 .^{2}$ We could follow these subjects for one year. All patients met the DSMIV-TR criteria. Table 1 shows characteristics of the cases. After the purpose and procedure of this study were fully explained, written informed consent was obtained from the all participants. This study was approved by the Ethics Committee of Nihon University.

During one-year follow-up, Global Assessment of Func- 
Table 1. Characteristics of cases

\begin{tabular}{|c|c|c|c|c|c|c|c|c|c|}
\hline \multirow{2}{*}{ Case numbers } & \multirow{2}{*}{ Diag } & \multirow{2}{*}{ Age (years) } & \multirow{2}{*}{ Sex } & \multirow{2}{*}{ Past drugs* (mg/day) } & \multicolumn{5}{|c|}{ BNS (mg/day) } \\
\hline & & & & & $\mathrm{BL}$ & $1 \mathrm{mo}$ & $3 \mathrm{mos}$ & $6 \mathrm{mos}$ & $12 \mathrm{mos}$ \\
\hline Case 1 & SZ & 21 & Female & OLZ (10) & 0 & 20 & 8 & 8 & 8 \\
\hline Case 2 & $\mathrm{SZ}$ & 41 & Female & PER (32) & 0 & 16 & 8 & $0^{\dagger}$ & 8 \\
\hline Case 3 & $\mathrm{SZ}$ & 34 & Female & OLZ (5) & 0 & 8 & 8 & 4 & 4 \\
\hline Case 4 & $\mathrm{SZ}$ & 40 & Female & Pimozide (4) & 0 & 12 & 12 & 12 & 8 \\
\hline Case 5 & $\mathrm{SZ}$ & 33 & Female & OLZ (7.5) & 0 & 16 & 16 & 16 & 12 \\
\hline Case 6 & SZ & 48 & Female & RIS (1) & 0 & 4 & 8 & 8 & 8 \\
\hline
\end{tabular}

*these are drugs before switching to blonanserin, tdata in case 2 were not used at 6 Ms because she did not have antipsychotics around 6 Ms. However, since we could follow her until endpoint, she was used in this study. Diag: diagnosis, SZ: schizophrenia, OLZ: olanzapine, PER: perospirone, RIS: risperidone, BNS: blonanserin, BL: baseline, mo: month

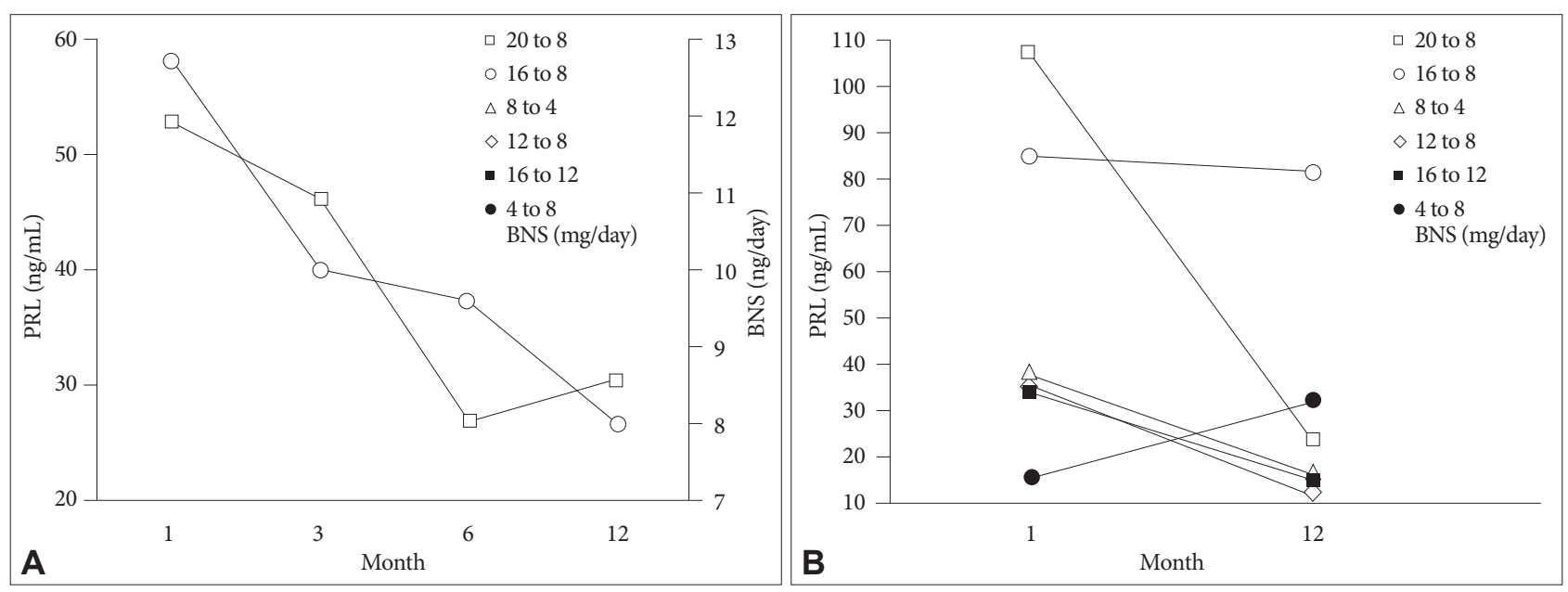

Figure 1. Blonanserin (BNS) dose and serum prolactin (PRL) level. A: The open square shows PRL; the open circle demonstrates BNS. Baseline was not shown because BNS is 0 . Data in case 2 were not used at 6 months because she did not have antipsychotics around 6 months (see Table 1). Thus, an accurate average dosage was according to five cases at 6 months. B: Six cases are shown: open square (case 1 in Table 1), open circle (case 2), open triangle (case 3), open rhomboid (case 4), closed square (case 5) and closed circle (case 6). BNS doses at 1 and 12 months of each case are illustrated in right side box (1 month to 12 months).

tioning (GAF), Positive and Negative Syndrome Scale (PANSS) and Drug-Induced Extrapyramidal Symptoms Scale (DIEPSS), participants' weight, total cholesterol, triglyceride, blood sugar, hemoglobin Alc and PRL were measured after switching to BNS. Details of study design were shown in our previous paper. ${ }^{2}$ We have presented data of the examination items except for GAF and PANSS in our previous study. In this study, PRL has been reanalyzed in more detail, and its data are shown. We considered that the number of cases of this study was unsuitable for statistical analyses. Therefore, no statistical analyses were done in this study.

Figure 1A shows averages of BNS doge and PRL level at 1 , 3,6 and 12 months (BNS doge: $12.7 \pm 5.9,10.0 \pm 3.3,9.6 \pm 4.6$ and $8.0 \pm 2.5$; PRL level: $52.8 \pm 35.1,46.1 \pm 18.7,26.9 \pm 11.9$ and 30.4 \pm 26.0 ). The mean PRL was increased from baseline (26.6 $\pm 18.4)$ to 1 month. Antipsychotics at baseline were olanzapine in three cases, and risperidone, perospirone and pimozide in one case each. As shown in Figure 1A, BNS dose was clearly associated with PRL level from 1 to 12 months. PRL was increased after switching to BNS; however, PRL was decreased depending on BNS dosages after 1 month. The average PRL level was almost normal when the mean BNS was $8.0 \mathrm{mg} /$ day (see at 12 months).

Figure 1B shows PRL levels of each patient (case 1-6 in Table 1 ) at 1 and 12 months. BNS dosages at 1 and 12 months of each patient are presented in right side box. PRL level was increased in only one case. However, her BNS dosage was increased (see closed circle case: case 6; 4 to $8 \mathrm{mg} /$ day). Although change of PRL was not remarkable in one case (open circle: case 2), PRL levels were decreased in all cases with reduction of BNS dosage.

In the follow-up, the mean BNS dosage was decreased, and BNS dosages of each case were also reduced except for the above one case (closed circle in Figure 1B). Regardless of the dose reduction, there were no remarkable changes from baseline to 12 months in PANSS and GAF (PANSS total score: 
$41.2 \pm 11.4$ to $37.5 \pm 8.9$; GAF: $72.0 \pm 23.4$ to $80.0 \pm 20.7)$. Moreover, no cases relapsed.

\section{DISCUSSION}

We switched to BNS from other antipsychotics within the same case, and then followed the case for one year. In the oneyear follow-up of BNS, it seemed that serum PRL level showed dose-dependent decrement. To our knowledge, the present study is the first report to examine dose dependency of PRL in long-term follow-up of the BNS treatment.

In general, the PRL raising of antipsychotic drugs seems to be dose dependent. ${ }^{4,6}$ In this study, that of BNS also appears to be dose dependent. Contrarily, RIS shows a high rate of hyperprolactinemia, and can influence PRL levels even at low doses. ${ }^{6}$ In previous studies, ${ }^{2,3}$ the PRL elevation was more frequently in RIS than in BNS. Taken together, BNS may be more suitable for schizophrenia maintenance treatment without hyperprolactinemia than RIS.

In our previous study, ${ }^{2}$ we were able to follow PRL levels for 3 months only in all cases because two cases had hyperprolactinemia, and stopped the BNS medications before 6 months. In that study, there were four cases switching to BNS from OLZ (OLZ-BNS case); their mean changes in PRL (baseline: 30.3 to 3 months: 64.0 ) was 33.7. Therefore, we concluded that hyperprolactinemia might be more often in BNS than in OLZ. In OLZ-BNS cases, one case relapsed, and stopped the BNS medications. Contrary to the comparison between baseline and 3 months, in the remaining three cases that we could follow for one year, the mean changes in PRL (baseline: 21.5 to 12 months: 14.7 ) was -6.8 . Mean dosages of BNS at 3 and 12 months were $12 \mathrm{mg}$ and $8 \mathrm{mg}$, respectively. On the basis of this finding, we can not state that hyperprolactinemia is more common in low dosage of BNS than in olanzapine. Thus, there may be little difference between low dosage BNS and olanzapine treatments in PRL rising.

In the maintenance treatment of schizophrenia, relapse is one of the most important issues in addition to side effects. We pay enough attention to relapse when drug dosage was decreased to avoid side effects. In this study, regardless of the dose decrease of BNS, there were no remarkable changes in symptoms and social functions. Moreover, no cases relapsed.

In conclusion, based on the possible characteristics of BNS recognized in our present and past studies, ${ }^{2}$ we consider that BNS, especially low dose BNS medication may be useful for schizophrenia maintenance treatment without hyperprolactinemia. Since PRL level was almost normal when the BNS dosage was $8 \mathrm{mg} /$ day, $8 \mathrm{mg}$ of BNS may be defined as the low dosage. However, since this study is a case series, the sample size was small; and no statistical analyses were performed. These may be potential limitations of the present study. Hence, findings of this study should be interpreted cautiously. In order to confirm the present results, further long-term comparative studies of BNS using larger samples are required.

\section{REFERENCES}

1. Deeks ED, Keating GM. Blonanserin: a review of its use in the management of schizophrenia. CNS Drugs 2010;24:65-84.

2. Takahashi S, Suzuki M, Uchiyama M. One-year follow-up study of psychotic patients treated with blonanserin: a case series. Asia Pac Psychiatry 2013;5:164-167.

3. Yang J, Bahk WM, Cho HS, Jeon YW, Jon DI, Jung HY, et al. Efficacy and tolerability of Blonanserin in the patients with schizophrenia: a randomized, double-blind, risperidone-compared trial. Clin Neuropharmacol 2010;33:169-175.

4. Haddad PM, Sharma SG. Adverse effects of atypical antipsychotics: differential risk and clinical implications. CNS Drugs 2007;21:911-936.

5. Henderson DC, Doraiswamy PM. Prolactin-related and metabolic adverse effects of atypical antipsychotic agents. J Clin Psychiatry 2008;69 (Suppl 1):32-44.

6. Walters J, Jones I. Clinical questions and uncertainty--prolactin measurement in patients with schizophrenia and bipolar disorder. J Psychopharmacol 2008;22:82-89. 